\title{
Comparaison de deux modèles cross-shore d'érosion des plages (Site de Rousty, Delta du Rhône, France)
}

\author{
David Morellato(a,b), François Sabatier(a,c), Frédéric Pons (d) et Pierre Gaufrès (b)
}

\author{
(a)CEREGE œ Centre Européen de Recherche et d'Enseignement en Géosciences de l'Environnement œ Europôle \\ de L'Arbois œ BP $80 \propto 13545$ Aix-en-Provence cedex 04, \\ Francemorellato@cerege.fr, sabatier@cerege.fr \\ (b)CETMEF o Bd du Président Kennedy -B.P. 543, 13092 AIX-EN-PROVENCE Cedex 02, \\ France Pierre.Gaufres@equipement.gouv.fr, David.Morellato@equipement.gouv.fr \\ (c)Delft University of Technology, Faculty of Civil Engineering, Hydraulic Engineering Section, \\ Stevinweg 1, 2628 CN Delft, The Netherlands. \\ (d)CETE Méditerranée, B.P. 37000, 13791 Aix-en-Provence Cedex3, \\ France Frederic.Pons@equipement.gouv.fr
}

\section{Résumé}

Nous avons comparé deux modèles numériques 2DV d'érosion des plages (SBEACH et LITPROF) sur une plage microtidale à barre du delta du Rhône. Les résultats montrent que le recul du rivage et l'allure morphologique de la berme sont relativement mieux simulés par SBEACH que par LITPROF. Par contre, aucun des deux modèles n'est capable de reproduire l'évolution sous-marine. Nous concluons sur l'intérêt de ces modèles pour simuler l'impact d'une tempête sur le recul du rivage et de la berme mais aussi sur 1'impossibilité de les utiliser pour des périodes de temps supérieures à un évènement de tempête.

\begin{abstract}
$\underline{\text { Abstract }}$
We compare two numerical models to simulate beach profile changes during storms on a microtidal barred beach on the Rhône delta: SBEACH and LITPACK. We conclude that SBEACH give better results to simulate berm retreat. Both model are not accurate to simulate bars movements.
\end{abstract}

Mots-clés : érosion, LITPROF, SBEACH, tempêtes, barres d'avant côte, berme, microtidal

\section{Introduction}

L'utilisation de modèles numériques est aujourd'hui une pratique courante en ingénierie côtière lorsqu'il s'agit de prévoir des processus d'érosion et/ou d'établir des scénarii de recalibrage et de rechargements artificiels des plages. Les modèles 2DV (bidimensionnel vertical) présentent 1'avantage d'être d'une utilisation aisée pour représenter de manière acceptable les processus des plages sableuses ouvertes à la houle. Les comparaisons de différents modèles 2DV sur les côtes Atlantiques américaines (Zheng et Dean, 1997) et des Pays-Bas (Van Rijn et al., 2003) montrent que si de nombreux modèles existent, il est encore aujourd'hui difficile de savoir, a priori, quel type de modèle est adapté pour telle ou telle plage. Ces modèles se divisent en deux catégories : des modèles semi-empiriques et des modèles de processus, dont la validité des résultats reste encore à améliorer. Notre objectif est donc de comparer les simulations de 2 modèles 2DV (SBEACH et LITPACK) de conception différente sur des mesures de profils de plage dans le delta du Rhône. 


\section{Méthodes}

\subsection{Acquisition des données morphologiques et des forçages climatiques}

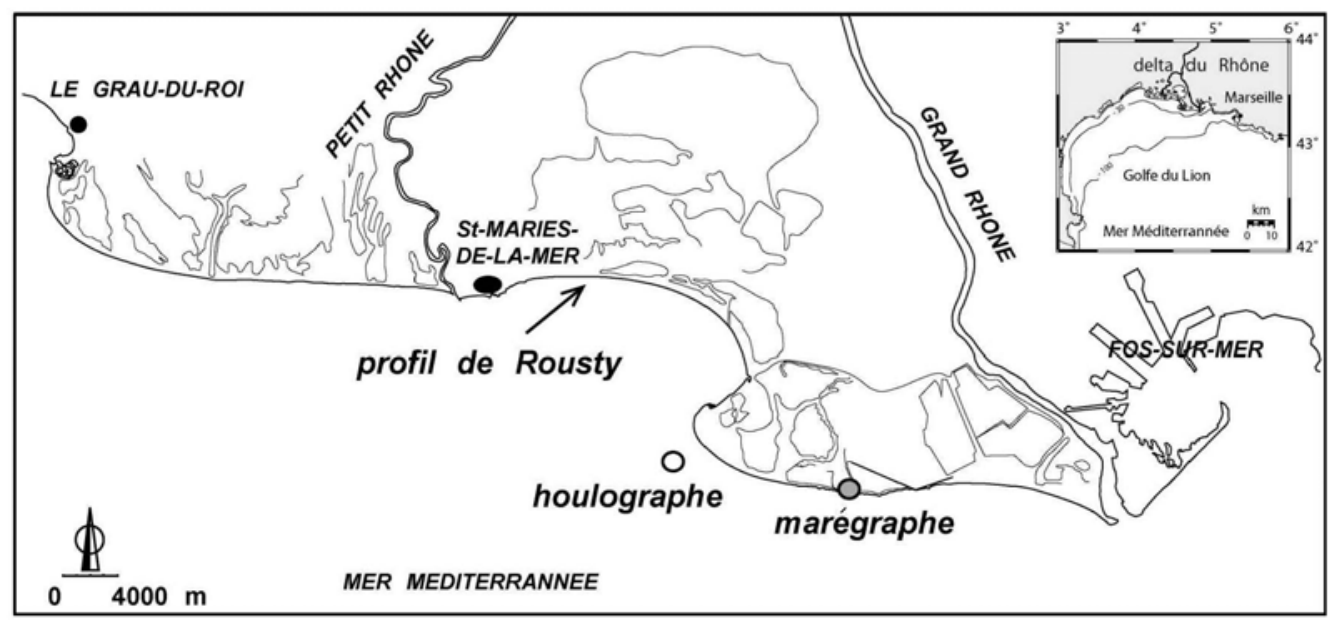

Figure 1:localisation géographique

Le profil de plage retenu (profil de Rousty, figure 1) est localisé dans une zone affectée d'un recul de 0 à œ5m.an . Cette plage à double barres a été choisie car elle montre peu de variations longitudinales, ce qui est idéal pour utiliser un modèle 2DV. Les relevés topo-bathymétriques de la plage ont été réalisés au moyen d'un tachéomètre électronique Leica (TC705) et d'un échosondeur couplé à un DGPS équipé sur une embarcation légère. Ils s'étendent sur un axe perpendiculaire au rivage depuis la dune jusqu'à la profondeur de fermeture (œ $6 \mathrm{~m}$ environ). Nous testons 3 profils de plages significatifs d'érosion durant une tempête ( 26 et 28 février et 17 mars 2001). Les houles incidentes prises en compte dans les modèles proviennent d'une bouéehoulographe DATAWELL de Port Gardian (figure 1, base de données CANDHIS, http://www.cetmef.equipement.gouv.fr). Les niveaux de la mer sont fournis par un courantomètre à pression $\mathrm{S} 4 \mathrm{ADW}$, à œ3 mètres de profondeur et à $0,3 \mathrm{~m}$ au-dessus du fond pour la période du 26 février au 3 mars 2001 et par un enregistrement automatique sur papier situé au grau de la Dent (Figure $\mathrm{n}^{\circ}$ 1) pour la période du 4 au 17 mars 2001. Les modèles montrant peu de sensibilité au vent, ce forçage $n$ 'est pas pris en compte.

\subsection{Les modèles utilisés et l'estimation de leur erreur}

Deux modèles numériques 2DV ont été utilisés : SBEACH, du CERC (Coastal Engineering Research Center) $^{5,6}$ et LITPROF, module cross-shore du modèle LITPACK du DHI (Danish Hydraulic Institute). Ces deux modèles appartiennent à des types différents : SBEACH est semiempirique et LITPACK intègre plus de processus. Ils sont tous les deux constitués de trois modules qui tournent de manière itérative au pas de temps choisi par l'utilisateur:

-Transformation des vagues à la côte. SBEACH utilise la théorie linéaire de la houle alors que LITPROF permet de choisir entre différentes théories dont la houle d'Isobe et Horikawa et les houles de Stokes de 1'ordre 1 à 5 .

-Calcul du taux de transport. La différence essentielle entre les deux modèles repose sur ce module. SBEACH calcule ce taux, en utilisant la différence entre le profil initial et le profil d'équilibre de forme exponentielle, atteint si les forçages sont les mêmes. 
LITPROF calcule ce taux en résolvant l'équation de diffusion verticale des sédiments en régime turbulent reliant la concentration des sédiments et le vecteur de diffusion. Le module LITSTP, utilisant cette équation, doit être lancé avant toute simulation sur LITPROF ; il lui fournit une matrice pour calculer ce taux de transport. Ce module permet d'intégrer le transit littoral mais celui-ci a été considéré comme nul dans nos simulations.

-Calcul du nouveau profil de plage à l'aide de l'équation de conservation de la masse.

Pour comparer les profils simulés avec les profils mesurés, nous avons testé les profils selon deux méthodes : -une observation visuelle, ou qualitative, en essayant de faire correspondre au mieux la forme du profil obtenu avec le profil final. -une quantification mathématique de l'erreur entre les profils mesurés et simulés, en essayant de minimiser le rapport $\mathrm{R}(\mathrm{X}){ }_{\sum_{\sum}}(\mathrm{Zi}-\mathrm{Zi}$

$R(x)=\sum_{i=0}^{x}\left(z i^{\text {mesuré }}-z i_{\text {calcule }}\right)$

où Zi représente la cote et X 1'abscisse du profil (nombre entier). Les résultats sont considérés comme bons, lorsque la valeur de $\mathrm{R}(\mathrm{X})$ est faible. La fonction $\mathrm{R} \mathrm{s}^{6}$ exprime en $\mathrm{m} \times$ et représente donc au point $X$ la différence entre les deux profils de 1'origine jusqu'à $X$.

\section{Résultats}

\subsection{Description des tempêtes et de leur impact morphologique}

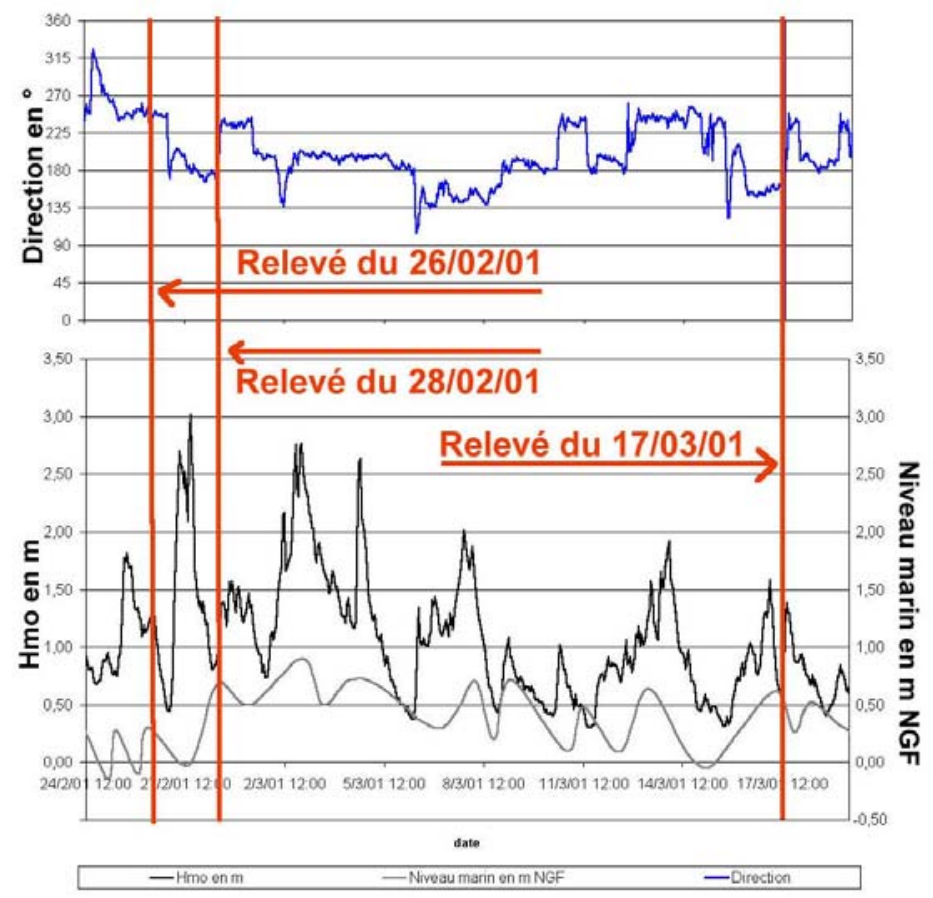

Figure 2: les conditions météo-marines 
La période entre le 26 et le 28 février est marquée par une tempête de 12 heures pendant lesquelles Hmo atteint 3 mètres et le niveau de la mer $+0,50 \mathrm{~m}$ NGF. La période entre le 28 février et le 17 mars est marquée par 6 tempêtes avec des hauteurs de houle dépassant 1,5m (2 ont une hauteur de 2,5m) entrecoupées d'accalmies. Le niveau maximal de la mer atteint $+0,90 \mathrm{~m}$ NGF. Nos relevés présentent l'intérêt d'encadrer parfaitement une tempête et une série de tempêtes afin d'évaluer les modèles dans deux situations érosives différentes.

Entre le 26/02/01 et le 28/02/01 (Figure 3), la bâche et la berme côté terre sont légèrement modifiées et la position du trait de côte reste pratiquement inchangée (recul de 19 centimètres). Par contre la pente de la berme du côté mer s'adoucit (pente de 5,5\% à 2,5\%) et une partie de ce

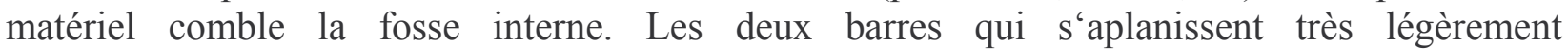

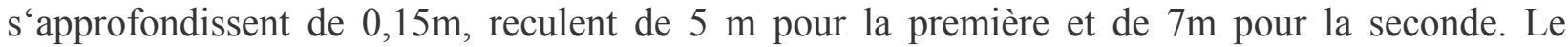
mouvement global des sédiments est donc un mouvement vers le large. Cependant, le profil du 28/02/01 est enrichi de $24 \mathrm{~m}^{1}$ (sur un mètre linéaire de plage) par rapport à celui du 26/02/01. Entre le 28 février et le 17 mars 2001, la bâche est comblée suite au recul de la berme qui

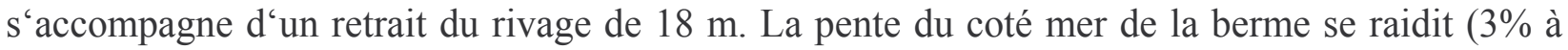
$8 \%$ ). Bien que les deux barres avancent ( $9 \mathrm{~m}$ pour la première et 11 mètres pour la seconde), elles montrent des comportements différents. La première $\mathrm{s}^{6}$ approfondit de $0,1 \mathrm{~m}$ et son allure demeure inchangée tandis que la seconde s'élève de $3 \mathrm{~cm}$ et se cambre. Toutefois la distance entre les barres et le rivage augmente de $9 \mathrm{~m}$ et $7 \mathrm{~m}$ respectivement pour la barre interne et externe. Le profil du 17/03/01 est érodé de $35 \mathrm{~m}^{1}$ (sur un mètre linéaire de plage) par rapport à celui du 28/02/01.

\subsection{Simulations des profils}

Les deux modèles ont subi des tests de sensibilité et de calage (Morellato, 2003) pour être finalement utilisés avec les valeurs des paramètres présentés dans le tableau 1.

Tableau 1: Paramètres retenus pour SBEACH et LITPROF

\begin{tabular}{|c|c|c|c|}
\hline \multicolumn{2}{|c|}{ SBEACH } & \multicolumn{2}{|c|}{ LITPROF } \\
\hline D50 & $0,16 \mathrm{~mm}$ & D50 & $0,16 \mathrm{~mm}$ \\
\hline $\mathrm{K}$ & $2,5.10-7 \mathrm{~m} 4 \mathrm{~N}-1$ & Porosité & 0,4 \\
\hline$\varepsilon$ & $0,002 \mathrm{~m} \times . \mathrm{s}-1$ & Densité & 2,65 \\
\hline$\kappa$ & 0,5 & Vitesse de chute & $0,022 \mathrm{~m} . \mathrm{s}-1$ \\
\hline $\begin{array}{c}\text { Température de } \\
\text { 1'eau }\end{array}$ & $11^{\circ} \mathrm{C}$ & Modèle de vague & Isobe et Horikawa \\
\hline $\begin{array}{c}\text { Angle maximal } \\
\text { d'avalanche }\end{array}$ & $30^{\circ}$ & $\gamma 1$ & 0,88 \\
\hline $\begin{array}{l}\text { Profondeur du } \\
\text { swash }\end{array}$ & $50 \mathrm{~cm}$ & $\gamma 2$ & 0,6 \\
\hline
\end{tabular}

Lors de la 1ère simulation (figure 3), la courbe d'erreur $\mathrm{R}(\mathrm{X})$ varie dans une fourchette de 0 à 9 (figure 5). $\mathrm{R}(\mathrm{x})$ est légèrement meilleur pour $\mathrm{SBEACH}$, jusqu'au niveau de la berme tandis que LITPACK est plus performant dans la partie sous-marine. L'allure du profil simulé par SBEACH est cependant plus cohérente avec les mesures. Lors de la 2nd simulation (figure 4), la courbe $d^{\prime}$ erreur $R(X)$ est sans conteste meilleure pour SBEACH que pour LITPROF mais cette dernière 
varie de 0 à 50, soit un facteur 5 par rapport à la simulation précédente (figure 5). Au niveau de la berme et de la fosse interne, les valeurs de $\mathrm{R}(\mathrm{X})$ de SBEACH sont toutefois similaires lors des deux simulations. LITPACK simule une pente trop abrupte. L'évolution sous-marine et plus particulièrement celle des barres est très mal reproduite. Seul LITPACK est capable de «créer » des barres mais de manière très différente des mesures.

Tableau 2: Résultat des simulations

\begin{tabular}{|c|c|c|c|c|}
\hline & Dune et avant plage & Berme & Barre interne & Barre externe \\
\hline \multicolumn{5}{|c|}{ Simulation entre le 26 et le 28 février 2001} \\
\hline Observations & $\begin{array}{l}\text { Légère projection de } \\
\text { sable sur 1'avant-plage }\end{array}$ & Erodée & Recul & Recul \\
\hline SBEACH & $\begin{array}{l}\text { Evolution non } \\
\text { reproduite }\end{array}$ & $\begin{array}{c}\text { Erodée de } \\
\text { façon } \\
\text { linéaire } \\
\end{array}$ & $\begin{array}{l}\text { Erodée mais pas } \\
\text { déplacée }\end{array}$ & $\begin{array}{l}\text { Evolution non } \\
\text { reproduite }\end{array}$ \\
\hline LITPROF & $\begin{array}{c}\text { Evolution non } \\
\text { reproduite }\end{array}$ & Erodée & $\begin{array}{c}\text { Evolution non } \\
\text { reproduite }\end{array}$ & $\begin{array}{c}\text { Evolution non } \\
\text { reproduite }\end{array}$ \\
\hline \multicolumn{5}{|c|}{ Simulation entre le 28 février et le 17 mars 2001} \\
\hline Observations & $\begin{array}{c}\text { Projection de sable sur } \\
\text { 1'avant-plage } \\
\end{array}$ & Erodée & $\begin{array}{c}\text { Avancée et } \\
\text { approfondissement }\end{array}$ & $\begin{array}{c}\text { Avancée et } \\
\text { élévation }\end{array}$ \\
\hline SBEACH & $\begin{array}{l}\text { Evolution non } \\
\text { reproduite }\end{array}$ & $\begin{array}{c}\text { Erodée de } \\
\text { façon } \\
\text { linéaire } \\
\end{array}$ & Supprimée & $\begin{array}{c}\text { Erodée mais pas } \\
\text { déplacée }\end{array}$ \\
\hline LITPROF & $\begin{array}{l}\text { Evolution non } \\
\text { reproduite }\end{array}$ & $\begin{array}{c}\text { Pas } \\
\text { suffisamment } \\
\text { érodée }\end{array}$ & \multicolumn{2}{|c|}{$\begin{array}{l}\text { Création d'un système à barres } \\
\text { multiples, différent de la réalité. }\end{array}$} \\
\hline
\end{tabular}

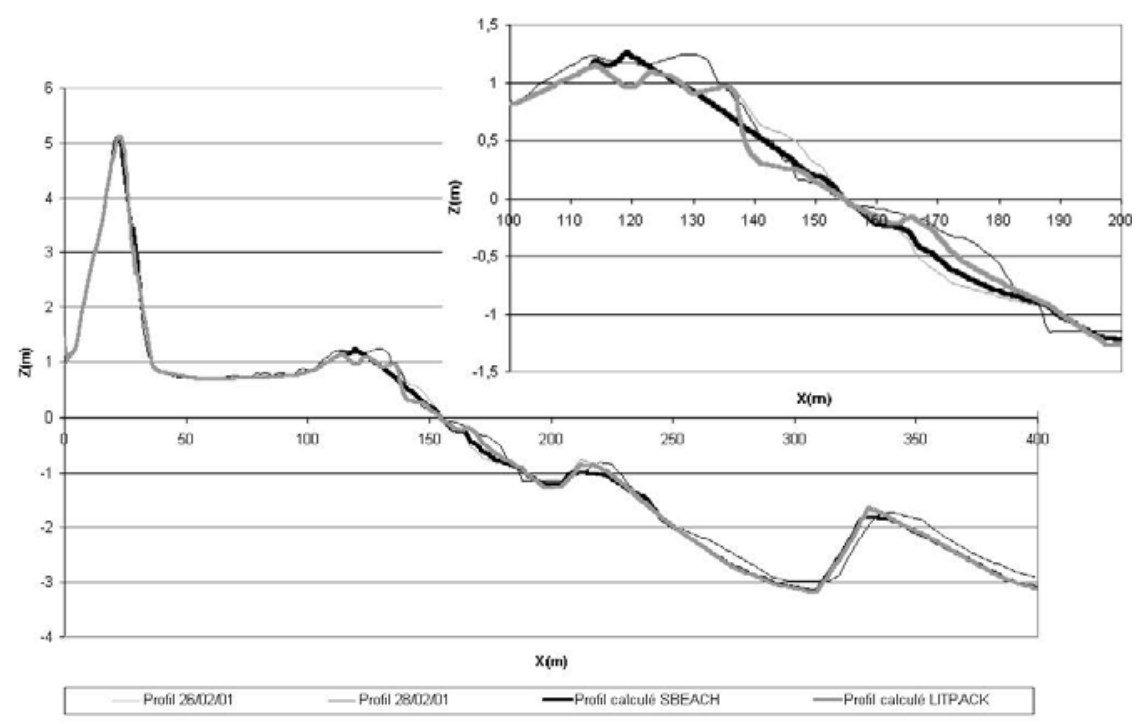

Figure 3: Comparaison des 2 modèles lors de la première simulation (26/02/01 au 28/02/01) 


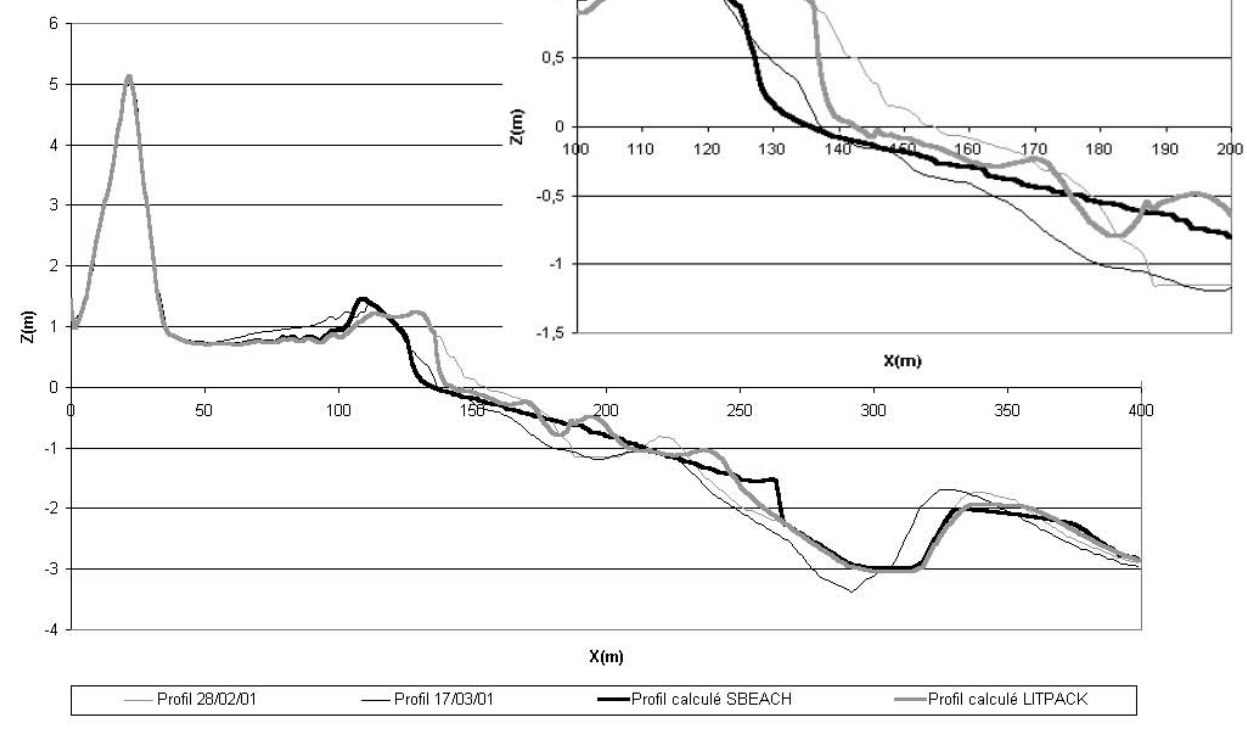

Figure 4: Comparaison des 2 modèles lors de la seconde simulation (28/02/01 au 17/03/01)
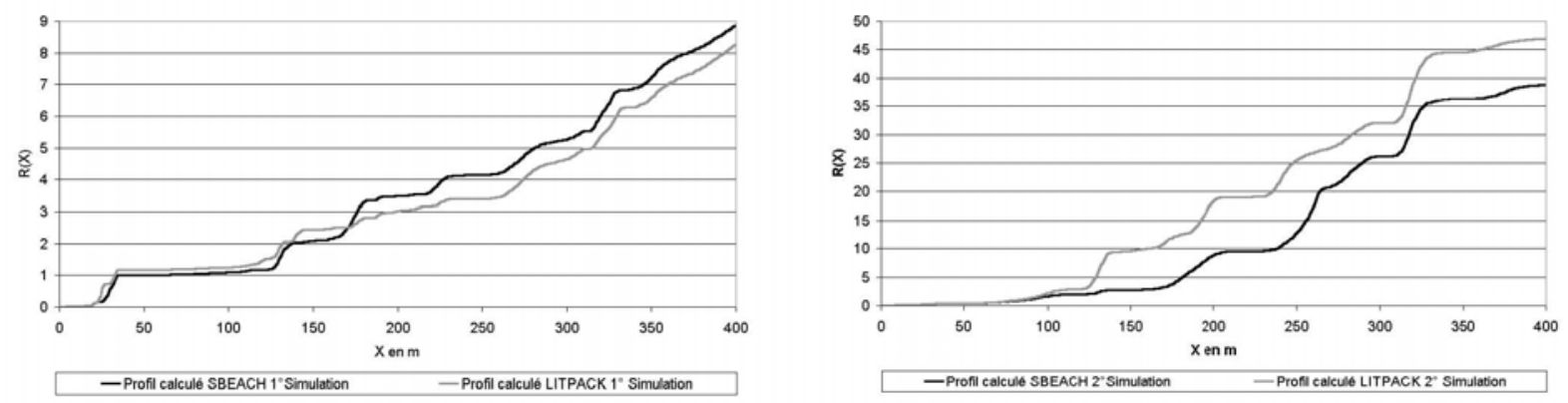

Figure 5: Evaluation de l'erreur des deux modèles

\section{Discussions}

SBEACH et LITPROF ne rendent pas compte de l'évolution du profil de plage entre la dune et le bourrelet car ces modèles ne considèrent pas le transport éolien, ni les processus de débordement de tempête et d'overwash. SBEACH est capable d'éroder la berme et d'élever sa crête mais il est incapable de distribuer les sédiments en arrière de la berme. Ces lacunes, et la difficulté du modèle à simuler des phases d'accrétion (Sabatier et al. ) ne permettent pas d'envisager des simulations supérieures à des événements de tempêtes. Les mauvais résultats de SBEACH en ce qui concerne la partie sous-marine étaient attendus car ce modèle n'est pas censé reproduire la morphodynamique sous-marine. Nos résultats divergent sur ce point de ceux de Zheng et Dean qui reproduisent l'évolution sous-marine d'une plage à Ocean City (Océan Atlantique) démontrant les différentes interprétations de la validité du modèle selon les sites d'études. 
La morphodynamique des plages à Ocean City est différente de celles du site de Rousty : les déplacements de barres sont plus réduits qu'en Camargue et la berme recule mois vite. De plus, SBEACH a été conçu et validé pour des houles plus longues que celles de Méditerranée ce qui induit probablement des biais lorsque ce modèle est utilisé dans des conditions différentes. Nos simulations montrent cependant de bons résultats au niveau de la berme. Par contre, les simulations mal reproduites de LITPROF sur les barres d'avant-côte sont surprenantes car ce modèle prétend simuler les fondsen ajoutant plus de complexité. Les différences entre les mesures et les calculs peuvent aussi provenir des relevés de terrain. Une plage n'est jamais complètement rectiligne et de petites modifications morphologiques pourraient avoir des répercussions importantes sur le modèle.

Pour pallier ce problème, la comparaison des modèles de Van Rijn et al. se base sur 10 profils moyennés (espacés de $100 \mathrm{~m}$ ) pour lisser la variabilité longitudinale. Cette approche n'est malheureusement pas possible dans notre cas car un seul suivi de profil est disponible, elle souligne cependant les difficultés et la nécessité d'obtenir des mesures de terrain nombreuses et fiables. Les biais entre les mesures de terrain et les simulations numériques proviennent aussi du fait que les modèles redistribuent les sédiments selon le principe de la conservation de la masse. Or le terrain indique que la réponse morphologique aux tempêtes peut être un gain ou une perte sédimentaire. Par définition, les deux modèles sont donc incapables de prendre en compte ce phénomène probablement dû au transport longitudinal et à des échanges sédimentaires avec les zones plus profondes. Nous sommes ici aux limites des modèles 2DV, car le site étudié présente des caractéristiques optimales pour ce type de modèle (plage rectiligne, houle perpendiculaire à la côte, courte durée de simulation). Enfin, un biais des modèles provient aussi probablement du fait que la granulométrie est supposée constante et égale au D50, alors que cet indice varie entre 0,25 et $0,16 \mathrm{~mm}$ sur le profil de Rousty. Choisir arbitrairement une granulométrie, n'a aucune signification physique car en réalité, les grains de taille différente se déplacent à des vitesses différentes, contrairement à ce que supposent nos deux modèles.

\section{$\underline{\text { 5.Conclusion }}$}

Sur le site de Rousty, la zone qui est convenablement reproduite pendant des événements de tempêtes par les deux modèles s'étend du bourrelet à la première fosse. SBEACH, modèle semiempirique, donc moins sophistiqué, donne paradoxalement de meilleurs résultats que LITPROF, modèle qui décrit plus finement les processus. SBEACH peut donc être utilisé dans le domaine de prévention des risques. En effet, il est possible de l'utiliser pour prévoir l'impact d'une tempête de projet sur le recul du rivage et de la berme et donc d'optimiser les projets de recalibrage de plage et de rechargement artificiel. Nos conclusions reposent cependant sur deux cas d'érosion, les recherches en cours visent à confirmer ces résultats à partir de plusieurs exemples le long des plages du Golfe du Lion.

\section{Remerciements}

Nous remercions DHI France pour le prêt du logiciel LITPACK pendant la période de travail et le CETMEF de Brest pour les données de houles. M. Chaibi,

A. Lambert, E. Mignot, et O. Samat sont remerciés pour leurs participations aux mesures de terrain. Ce travail fait partie des programmes RESYST et LITEAU financé par le MATE. 


\section{Bibliographie}

1.Zheng J. et Dean R.G., Numericals models and intercomparisons of beach profile evolution. Coastal Engineering 30, 1997, P. 169-201.

2.Van Rijn L.C., Walstra D.J.R., Grasmeijer B., Sutherland J., Pan S., Sierra J.P. The predictability of cross-shore bed evolution of sandy beaches at the time scale of storms and seasons using process-bases Profile models. Coastal Engineering 47, 2003, P. 295-327.

3.Sabatier F. Fonctionnement et dynamiques morphosédimentaires du littoral du delta du Rhône. Thèse, Université Aix-Marseille III, 2001, 272p.

4.Morellato D., Modèles numériques de transport solide littoral dans le profil -Application de Sbeach et Litpack aux profils du programme Liteau en Camargue, Travail de Fin d'Etude, Ecole Nationale des Travaux Publics de l'Etat, 2003, 141p.

5.Larson M., Kraus N .C. SBEACH : Numerical model for simulating storm-induces beach change, Report 1, empirical fondation and model developement. CERC œ WES, Technical Report CERC-89-9, 1989, 267p.

6.Larson M., Kraus N. C.,Byrnes M. R. SBEACH : Numerical model for simulating storminduces beach change, Report 2, Numerical formulation and model tests. CERC œ WES, Technical Report CERC-89-9, 1990, 121p.

7.DHI . LITPACK : An integrated Modeling System for LIToral Processes And Coastline Kinetics, Short Introduction and Tutorial. DHI Software, 2001, 95p

8.Sabatier F., Lambert A., Chaibi M., Samat O., Provansal M. Morphodynamique du profil de plage en milieu microtidal : du relevé de terrain au modèle mathématique et numérique. Geomophology : from expert opinion to modelling, 2002, P.356-360. 